\title{
Sharbat: An Important Dosage Form of Unani System of Medicine
}

\author{
Imtiyaz AHMAD ${ }^{1}$, Shariq SHAMSI ${ }^{1}$, Roohi ZAMAN ${ }^{1}$
}

${ }^{1}$ Department of IImul Saidla (Unani Pharmacy), National Institute of Unani Medicine, Bengaluru, India.

\section{ABSTRACT}

The present study was designed to discuss many important points related to Sharbat with different types of drugs (juicy fruits, dried sour fruits, dried sweet fruits, dry herbal drugs, mucilaginous drugs, drugs having volatile constituents, and so forth). It also aimed to extensively discuss the drug and water ratio and drug and sugar ratio to prepare Sharbat, which is mentioned in different reference books of Unani system of medicine (USM). USM includes many safe and effective single drugs as well as compound formulations of herbal, animal, and mineral origin, which are used to cure a wide range of diseases. Unani compound preparations are commonly used in four forms: solid (Hab, Qurs, Safoof, Kushta, and so forth); semisolid (Majoon, Laooq, Marham/Zamad, and so forth); liquid (Sharbat, Sheera, Rooh, Tila, and so forth); and gaseous (Bakhoor, Inkabab, and so forth). Sharbat is an important dosage form used from ancient times. It is mostly used for the respiratory, urinary, digestive, and cardiovascular systems of the body.

Key words: Dosage form, Unani system of medicine, Sharbat.

\section{INTRODUCTION}

The word Sharbat (English, sherbet) is a soft drink or liquor that incorporates white sugar, misri, honey, and gur dissolved in water. However, in the Greco-Arab pharmacopeia, Sharbat implies a concentrated liquor made from decoction or fruit juices by adding sugar to yield a qiwam (1). Sharbat (syrup) is made by preparing the decoction or infusion of the ingredient drugs or by taking the juice of the fruit, which is then mixed with sugar and boiled to a required consistency (2). The invention of Sharbat is attributed to Pythagoras (an ancient Greek philosopher and mathematician). Sharbat is characterized by its sweet taste (i.e., good palatability). The reason behind the innovation of Sharbat dosage form lies in the fact that most of the herbs, if taken raw, are highly impalatable. So, to enhance palatability and prolong the storage period of medicines, Sharbat dosage form was designed in the Unani system of medicine (USM) (1). The equivalent of Sharbat in a modern system is syrup. Syrups are concentrated, viscous, aqueous solutions of sugar substitute with or without flavors and medical substances (3). The syrup is used to treat coughs and sore throats, relieve tickling and irritation of the throat, loosen phlegm, facilitate expectoration, and heal and soothe the throat and lungs (4). When purified water alone is used in making the solution of sucrose, the preparation is known as syrup or simple syrup if the sucrose concentration is $85 \%(3)$.

\section{SURVEY OF LITERATURE}

The word Sharbat is derived from a Persian term "sharbat" and the word Sherbet is derived from a Turkish term "şerbet" "sherbet," both of which in turn originate from an Arabic term "sharba," a drink from "shariba" to drink. It is also known as "sorbet," in French "sorbet," in Italian "sorbetto," and in Turkish "şerbet." The word is equivalent to syrup in both American and British English. In olden times, it was an iced fruit soft drink or a cool effervescent. The spelling, meaning, and pronunciation are different in different countries. It is usually spelled as "sherbet," but some people wrongly spell 
it as "sherbert" (5). The origin of Sharbat is buried in antiquity. Traditionally its invention has been attributed to Pythagoras, the ancient Greek philosopher and mathematician $(1,6)$. The incorporation of various ingredients into a Sharbat prolongs the life of medicinal ingredients. Also, due to the palatability of Sharbat, the patient is not reluctant to drink it. However, it must be born in mind that the Sharbat described here serves as a vehicle for appropriate medicines prescribed for the ailments (1).

Sharbat is a popular drink in West and South Asia prepared from fruits or flower petals. It is sweet in taste. Popular Ashriba is made of one or more of the following: rose water, sandalwood, bael, gurhal, lemon, orange, mango, pineapple, and falsa. In the twelfth century, Ismail Jurjani mentioned in his book Zakhirah Khwarazmshahi different types of Ashriba in Iran, including gur, anar, sekanjebin, and so forth (7).

It was popularized in the Indian subcontinent by Babar, who sent for frequent loads of ice from the Himalayas to make a cool refreshing drink. Spices and fruits to be used in Sharbat were grown in the gardens of the Ottoman Palace under the control of pharmacists and doctors of the Palace (5).

Sharbat is a sweet aqueous liquid made by dissolving sugar, misri, honey, or jaggery in water. But according to Qarabadeen [such as National Formulary of Unani Medicine Part-I (NFUM-I), Al-qarabadeen, Bayaz kabeer, Qarabadeen majeedi, Qarabadeen jadeed], Sharbat is a sweet viscous liquid made by preparing the decoction or infusion of the ingredient drugs or by taking out the juice of the fruit which is then mixed with sugar and boiled to a required consistency (qiwam), that is of one $\operatorname{tar}(2,6,8-10)$.

The decoction or infusion of ingredient drugs or juice of fruits is poured into a coated vessel, and 2.5 or 3 parts of sugar is added. Then, the vessel is kept on a low fire and boiled till the required consistency is obtained. To test the qiwam of Sharbat, it is first pressed between the thumb and the index finger and observed for one tar, or a drop of the Sharbat is put on the floor. If the drop does not spread on the floor, it is considered to be the required giwam (1). In USM, various types of Ashriba are used. The methodology of the preparation of Sharbat is different for different types of drugs. For example, the methodology is different for juicy fruits, dried sour fruits, dried sweet fruits, dry herbal drugs, mucilaginous drugs, volatile constituents, and so forth.
Methodology of preparation of Sharbat of different drugs in USM

1. Preparation of Sharbat of "juicy fruits" such as grape, pomegranate, apple, and so forth

Sharbat from juicy fruits: The juice of the fruit is obtained, mixed with 2.5 or 3 times of sugar, and boiled on a low fire to the required consistency of Sharbat (1).

\section{Preparation of Sharbat of "dried sour fruits" such as imli, zarishk, alu bukhara, and so forth}

The fruits are thoroughly washed and soaked overnight in water, amounting to six times the weight of the fruits. Next morning, the fruits are boiled and allowed to cool. They are then rubbed with hands and filtered through a piece of fine cloth. The filtered liquid is kept undisturbed for some time so that the heavier matter settles down at the bottom. Thereafter, the lighter liquid part is decanted in a vessel. A required quantity of sugar is added, and the mixture is boiled on a low fire to the required consistency. It is now filtered again through a piece of fine cloth to obtain the Sharbat (2).

\section{Preparation of Sharbat of "dried sweet fruits" such as unnab, anjeer, and so forth}

The fruits are thoroughly washed and soaked overnight in water, amounting to six times the weight of the fruits. Next morning, the fruits are boiled, allowed to cool, and then filtered through a piece of fine cloth. A required quantity of sugar is added, and the mixture is boiled on a low fire to obtain the desired consistency of Sharbat (8).

\section{Preparation of Sharbat of "dry herbal drugs"}

Dry herbal drugs are soaked overnight in water, amounting to 8 or 10 times the weight of the drugs. Next morning, they are boiled till one third water is left, allowed to cool, rubbed with hands, and filtered through a piece of fine cloth. Then, two or three times of sugar is added, and the mixture is boiled on a low fire to obtain the desired consistency of Sharbat (8).

\section{Preparation of Sharbat with "mucilaginous drugs" such as behidana, sapistan, and so forth}

When the mucilaginous drugs are used as ingredients in a particular formula along with other drugs, the Sharbat of the other drugs is prepared first and then mixed with the mucilaginous drugs. The boiling is continued till the mixture reaches its required consistency of Sharbat (2). 
6. Preparation of Sharbat with drugs having "volatile constituents" such as mushk, ambar, and so forth

These drugs are dissolved in any other specific arq. They are added in the last stage of the Sharbat preparation and mixed thoroughly (2).

\section{Preparation of Sharbat with turanjabeen}

Turanjabeen is dissolved in the decoction of other drugs and strained. The mixture is then decanted, and sugar is added three times the weight of the solution to make the Sharbat (2).

The methodology discussed earlier is a general methodology, but in different Unani books, different drug and water and drug and sugar ratios are recommended. Even in certain cases, the same type of Sharbat is prepared with different drug and water and drug and sugar ratios by Unani physicians. Therefore, it is important to summarize all the different variables used by Unani physicians while preparing Sharbat. Data on Sharbat mentioned in some important Unani books are given in Table 1.

\section{CONCLUSION}

This study described the preparation of an important dosage form Sharbat. Data were collected from the important reference books of USM. Hence this study might serve as a reference for preparing different Ashriba.

\section{ACKNOWLEDGMENT}

The authors owe their gratitude to Prof. Mansoor Ahmad Siddiqi, Director, National Institute of Unani Medicine, Bengaluru, for providing an academic research environment to work with excellence. They also thank the Library staff of the institute for providing necessary books for this study.

\begin{tabular}{|c|c|c|c|c|c|c|c|}
\hline Name of the Sharbat & $\begin{array}{l}\text { Drug } \\
\text { (g) }\end{array}$ & $\begin{array}{l}\text { Water } \\
(\mathrm{mL})\end{array}$ & $\begin{array}{l}\text { Sugar } \\
\text { (g) }\end{array}$ & $\begin{array}{l}\mathrm{D} \& W \\
\text { ratio }\end{array}$ & $\begin{array}{l}\mathrm{D} \& S \\
\text { ratio }\end{array}$ & $\begin{array}{l}\text { Yield of } \\
\text { Joshanda }\end{array}$ & $\begin{array}{c}\text { Therapeutic } \\
\text { Use/Medicinal Actions }\end{array}$ \\
\hline Sharbat Aloo baloo (12) & 240 & 960 & 960 & $1: 4$ & $1: 4$ & $1 / 2$ & \multirow{3}{*}{$\begin{array}{l}\text { Hisat-e-kulya (nephrolithiasis), Hisat-e-masana } \\
\text { (bladder stone), Mudirr-e-bol (diuretic) (10-12) }\end{array}$} \\
\hline Sharbat Aloo baloo (10) & 450 & 1800 & 1800 & $1: 4$ & $1: 4$ & $1 / 3$ & \\
\hline Sharbat Aloo baloo (11) & 500 & 2000 & 2000 & $1: 4$ & $1: 4$ & - & \\
\hline Sharbat Unnab (12) & 480 & 1920 & 1440 & $1: 4$ & $1: 3$ & - & \multirow{4}{*}{$\begin{array}{l}\text { Sual (cough), Waj'-al-sadar (chest pain), Fasad-e- } \\
\text { khoon (putrefaction of blood) }(2,10-12)\end{array}$} \\
\hline Sharbat Unnab (11) & 500 & 4000 & 1500 & $1: 8$ & $1: 3$ & - & \\
\hline Sharbat Unnab (10) & 450 & 1800 & 1350 & $1: 4$ & $1: 3$ & - & \\
\hline Sharbat Unnab (2) & 500 & - & 1500 & - & $1: 3$ & - & \\
\hline Sharbat Anar Sheereen (11) & - & $\begin{array}{c}900 \\
\text { (Aab anar) }\end{array}$ & 900 & $1: 1$ & $1: 1$ & $1 / 2$ & \multirow{3}{*}{$\begin{array}{l}\text { Ghasiyan (nausea), Atash-e-mufrit (excessive thirst), } \\
\text { Zof-e-qalb wa jigar (weakness of heart and liver) } \\
(2,10,11)\end{array}$} \\
\hline Sharbat Anar Sheereen (10) & - & $\begin{array}{c}900 \\
\text { (Aab anar) }\end{array}$ & 900 & $1: 1$ & $1: 1$ & $1 / 2$ & \\
\hline Sharbat Anar Sheereen (2) & - & $\begin{array}{c}1000 \\
\text { (Aab anar) }\end{array}$ & 1000 & $1: 1$ & $1: 1$ & - & \\
\hline Sharbat Anar Tursh (11) & - & $\begin{array}{c}1800 \\
\text { (Aab anar) }\end{array}$ & 900 & $1: 2$ & $1: 2$ & - & \multirow[t]{2}{*}{$\begin{array}{l}\text { Ghasiyan (nausea), Qai (vomiting), Zof-e-qalb } \\
\text { (weakness of heart) }(10,11)\end{array}$} \\
\hline Sharbat Anar Tursh (10) & - & $\begin{array}{c}1846 \\
\text { (Aab anar) }\end{array}$ & 900 & $1: 2$ & $1: 2$ & - & \\
\hline Sharbat Anjbar (12) & 85 & - & 480 & - & $1: 5$ & - & \multirow{4}{*}{$\begin{array}{l}\text { Nafs-ud-dam (hemoptysis), Kasrat-e-tams } \\
\text { (menorrhagia), Sahaj-e-am'a (enteritis), Ishal- } \\
\text { e-damwi (bloody diarrhea), Nazf-ud-dam } \\
\text { (hemorrhage), Zof-e-qalb wa me'da, (weakness of } \\
\text { heart and stomach) }(2,10,11,12 \text { ) }\end{array}$} \\
\hline Sharbat Anjbar (11) & 77 & - & 500 & - & $1: 6$ & - & \\
\hline Sharbat Anjbar (10) & 79 & - & 450 & - & $1: 5.5$ & - & \\
\hline Sharbat Anjbar (2) & 90 & - & 500 & - & $1: 5$ & - & \\
\hline Sharbat Banafsha (12) & 585 & 2400 & 960 & $1: 4$ & $1: 1.5$ & $1 / 3$ & \multirow{4}{*}{$\begin{array}{l}\text { Suda'(headache), Sual (cough), Nazla (coryza), } \\
\text { Humma (fever),Qabz (constipation), Waj-al-kulya } \\
\text { (renal pain) }(2,10,11,12 \text { ) }\end{array}$} \\
\hline Sharbat Banafsha (11) & 120 & - & 960 & $1: 8$ & - & - & \\
\hline Sharbat Banafsha (10) & 120 & - & 900 & - & $1: 7$ & - & \\
\hline Sharbat Banafsha (2) & 125 & - & 1000 & - & $1: 8$ & - & \\
\hline
\end{tabular}


TABLE 1: Continue.

\begin{tabular}{|c|c|c|c|c|c|c|c|}
\hline Name of the Sharbat & $\begin{array}{l}\text { Drug } \\
\text { (g) }\end{array}$ & $\begin{array}{l}\text { Water } \\
(\mathrm{mL})\end{array}$ & $\begin{array}{l}\text { Sugar } \\
\text { (g) }\end{array}$ & $\begin{array}{l}\mathrm{D} \& \mathrm{~W} \\
\text { ratio }\end{array}$ & $\begin{array}{l}\text { D\&S } \\
\text { ratio }\end{array}$ & $\begin{array}{l}\text { Yield of } \\
\text { Joshanda }\end{array}$ & $\begin{array}{c}\text { Therapeutic } \\
\text { Use/Medicinal Actions }\end{array}$ \\
\hline Sharbat Bazoori Barid (12) & 88 & 1440 & 940 & $1: 16$ & $1: 10$ & $1 / 2$ & \multirow{3}{*}{$\begin{array}{l}\text { Hiddat-e-jigar (hotness of liver), } \\
\text { Haar-wa-haad humma (acute fever), } \\
\text { Mudirr-e-bol (diuretic) }(10,11,12)\end{array}$} \\
\hline Sharbat Bazoori Barid (11) & 86 & - & 960 & - & $1: 11$ & - & \\
\hline Sharbat Bazoori Barid (10) & 86 & - & 900 & - & $1: 9$ & - & \\
\hline Sharbat Bazoori Motdil (11) & 35 & - & 60 & - & $1: 2$ & - & \multirow{3}{*}{$\begin{array}{l}\text { Humma-e-murakkaba (compound fever), } \\
\text { Ehtebas-e-tams (amenorrhea), Ehtebas-e-baul } \\
\text { (anuria), Tasaddud-e-kabid (hepatic obstruction) } \\
(2,10,11)\end{array}$} \\
\hline Sharbat Bazoori Motdil (10) & 35 & - & 60 & - & $1: 2$ & - & \\
\hline Sharbat Bazoori Motdil (2) & 400 & - & 550 & - & $1: 1.5$ & - & \\
\hline Sharbat Bazoori Har (11) & 375 & 1500 & 1000 & $1: 4$ & $1: 2.5$ & - & \multirow{2}{*}{$\begin{array}{l}\text { Buroodat-e-gurda wa jigar (coldness of kidney } \\
\text { and liver) }(10,11)\end{array}$} \\
\hline Sharbat Bazoori Har (10) & 330 & 1350 & 900 & $1: 4$ & $1: 3$ & - & \\
\hline Sharbat Deenar (11) & 490 & - & 1000 & - & $1: 2$ & - & \multirow{3}{*}{$\begin{array}{l}\text { Waqram-e-kabid (hepatitis), Waram-e-rahem } \\
\text { (uteritis), Yarqan-e-suddi (obstructive jaundice), } \\
\text { Istisqa (ascites), Zat-ul-janb (pleurisy), Qabz } \\
\text { (constipation) }(2,10,11 \text { ) }\end{array}$} \\
\hline Sharbat Deenar (10) & 442 & - & 900 & - & $1: 2$ & - & \\
\hline Sharbat Deenar (2) & 590 & - & 1200 & - & $1: 2$ & - & \\
\hline Sharbat Ejaz (11) & 765 & - & 1000 & - & $1: 1.5$ & - & \multirow{3}{*}{$\begin{array}{l}\text { Sual Yabis (dry cough), Nazla-o-Zukam (coryza } \\
\text { and catarrh), Sil-wa-diq (tuberculosis) }(2,10,11)\end{array}$} \\
\hline Sharbat Ejaz (10) & 628 & - & 900 & - & $1: 1.5$ & - & \\
\hline Sharbat Ejaz (2) & 765 & - & 1000 & - & $1: 1.5$ & - & \\
\hline Sharbat Khashkhash (11) & 1000 & - & 2000 & - & $1: 2$ & - & \multirow{3}{*}{$\begin{array}{l}\text { Sahar (insomnia), Nazla (coryza), Sual (cough), } \\
\text { Zaheer (dysentery), Is-hal (diarrhea) }(2,10,11)\end{array}$} \\
\hline Sharbat Khashkhash (10) & 900 & - & 1800 & - & $1: 2$ & - & \\
\hline Sharbat Khashkhash (2) & 1000 & - & 2000 & - & $1: 2$ & - & \\
\hline Sharbat Neelofar (12) & 30 & 204 & 900 & $1: 7$ & - & - & \multirow{3}{*}{$\begin{array}{l}\text { Atash-e-mufrit (excessive thirst), } \\
\text { Zof-e-qalb (weakness of heart), } \\
\text { Da'f-e-hiddat-e-sufra (bile palliative) }(2,11,12 \text { ) }\end{array}$} \\
\hline Sharbat Neelofar (11) & 120 & - & 960 & - & $1: 8$ & - & \\
\hline Sharbat Neelofar (2) & 125 & - & 1000 & - & $1: 8$ & - & \\
\hline Sharbat Tamarhindi (12) & 500 & - & 1000 & - & $1: 2$ & - & \multirow{3}{*}{$\begin{array}{l}\text { Da'f-e-hiddat-e-sufra, (bile palliative) Qabz } \\
\text { (constipation), Qai (vomiting), } \\
\text { Zof-e-me'da (weakness of stomach) }(2,11,12)\end{array}$} \\
\hline Sharbat Tamarhindi (11) & 500 & - & 1000 & - & $1: 2$ & - & \\
\hline SharbatTamar Hindi (10) & 450 & - & 900 & - & $1: 2$ & - & \\
\hline Sharbat Toot Siyah (11) & - & 1000 & 1500 & - & $1: 1.5$ & - & \multirow{3}{*}{$\begin{array}{l}\text { Dard wa warm-e-halaq (pharyngeal pain), } \\
\text { Khushoonat-e-halaq (sore throat) }(2,10,11)\end{array}$} \\
\hline Sharbat Toot Siyah (10) & 900 & - & 1350 & - & $1: 1.5$ & - & \\
\hline Sharbat Toot Siyah (2) & - & 1000 & 1500 & - & $1: 1.5$ & - & \\
\hline Sharbat Sadar (11) & 168 & - & 960 & - & $1: 6$ & - & \multirow{2}{*}{$\begin{array}{l}\text { Sual (cough), Nazla muzmin (chronic coryza), } \\
\text { Zeeq-un-nafas (asthma), Sil (pthysis) }(2,11)\end{array}$} \\
\hline Sharbat Sadar (2) & 2090 & - & 5000 & - & $1: 2.5$ & - & \\
\hline Sharbat Ahmad Shahi (11) & 114 & - & 225 & - & $1: 2$ & - & \multirow{2}{*}{$\begin{array}{l}\text { Malankhuliya } \\
\text { (melancholia) }(10,11)\end{array}$} \\
\hline Sharbat Ahmad Shahi (10) & 114 & - & 225 & - & $1: 2$ & - & \\
\hline Sharbat Falsa (11) & - & 500 & 1000 & - & $1: 2$ & - & \multirow{2}{*}{$\begin{array}{l}\text { Atash-e-mufrit (excessive thirst), Zof-e-qalb } \\
\text { wa me'da (weakness of heart and stomach), } \\
\text { Musakkin-e-harat-e-jigar (anodyne) }(10,11 \text { ) }\end{array}$} \\
\hline Sharbat Falsa (10) & 750 & 750 & 900 & $1: 1$ & $1: 1.25$ & - & \\
\hline Sharbat Sandal (11) & 168 & - & 900 & - & $1: 5.5$ & $1 / 2$ & \multirow{2}{*}{$\begin{array}{l}\text { Ghabrahat (anxiety), Khafqan (palpitation) Daf'e } \\
\text { hararat-e jigar wa me'da, (anodyne) }(10,11)\end{array}$} \\
\hline Sharbat Sandal (10) & 120 & 450 & 900 & $1: 4$ & $1: 7.5$ & $1 / 2$ & \\
\hline Sharbat Faryadars (11) & 198 & - & 1000 & - & $1: 5$ & - & \multirow[b]{2}{*}{ Sual (cough), Nazla (coryza) $(2,11)$} \\
\hline Sharbat Faryad ras (2) & 1415 & - & 9000 & - & $1: 6$ & - & \\
\hline Sharbat Anjeer (11) & 225 & - & 750 & - & $1: 3$ & - & \multirow{2}{*}{$\begin{array}{l}\text { Qabz (constipation), Daf'e warm-e-tihal } \\
\text { (splenitis) }(10,11)\end{array}$} \\
\hline Sharbat Anjeer (10) & 225 & - & 750 & - & $1: 3$ & - & \\
\hline SharbatAngoor shireen (11) & - & $\begin{array}{c}900 \\
\text { (Aab angoor) }\end{array}$ & 1350 & - & $1: 1.5$ & - & \multirow[t]{2}{*}{$\begin{array}{l}\text { Zo'f-e-bah (anaphrodisia), Zo'f-e-am (general } \\
\text { weakness) }(10,11)\end{array}$} \\
\hline SharbatAngoor shireen (10) & - & $\begin{array}{c}900 \\
\text { (Aab angoor) }\end{array}$ & 2700 & - & $1: 3$ & - & \\
\hline
\end{tabular}


TABLE 1: Continue.

\begin{tabular}{|c|c|c|c|c|c|c|c|}
\hline Name of the Sharbat & $\begin{array}{l}\text { Drug } \\
\text { (g) }\end{array}$ & $\begin{array}{l}\text { Water } \\
(\mathrm{mL})\end{array}$ & $\begin{array}{l}\text { Sugar } \\
\text { (g) }\end{array}$ & $\begin{array}{l}\mathrm{D} \& \mathrm{~W} \\
\text { ratio }\end{array}$ & $\begin{array}{l}\mathrm{D} \& S \\
\text { ratio }\end{array}$ & $\begin{array}{l}\text { Yield of } \\
\text { Joshanda }\end{array}$ & $\begin{array}{l}\text { Therapeutic } \\
\text { Use/Medicinal Actions }\end{array}$ \\
\hline Sharbat Fawakeh (11) & - & $\begin{array}{c}6300 \\
\text { (Aab fawakeh) }\end{array}$ & 2700 & - & - & - & \multirow[t]{2}{*}{$\begin{array}{l}\text { Zo'f-e-a'za-e-raeesa (weakness of vital organs), } \\
\text { Zo'f-e-am (general weakness }(10,11)\end{array}$} \\
\hline Sharbat Fawakeh (10) & - & 2700 & 1800 & - & - & $1 / 2$ & \\
\hline Sharbat Kasoos (12) & 148 & - & 405 & - & $1: 3$ & - & \multirow{2}{*}{$\begin{array}{l}\text { Humma-e-murakkaba (compound fever) } \\
\text { Zof-e-jigar wa me'da (weakness of liver and } \\
\text { stomach) }(11,12)\end{array}$} \\
\hline Sharbat Kasoos (11) & 125 & - & 408 & - & $1: 3$ & - & \\
\hline Sharbat Rangatra (11) & 1000 & - & 1000 & - & $1: 1$ & - & \multirow{2}{*}{$\begin{array}{l}\text { Daf'-e-hiddat-e-sufra (bile palliative), Atash-e- } \\
\text { mufrit (excessive thirst) }(10,11)\end{array}$} \\
\hline Sharbat Rangatra (10) & 225 & - & 900 & - & 1:3.5 & - & \\
\hline Sharbat Zufa Sada (11) & 250 & - & 2000 & - & $1: 8$ & - & \multirow[t]{2}{*}{ Zeeq-un-nafas (asthma), Sual (cough) $(10,11)$} \\
\hline Sharbat Zoofa Sada (10) & 250 & - & 2250 & - & 1:9 & - & \\
\hline Sharbat seb Sheereen (12) & - & $\begin{array}{c}9600 \\
\text { (Aab seb) }\end{array}$ & 960 & - & - & - & \multirow{3}{*}{$\begin{array}{l}\text { Da'f-e-ishal sufrawi, (antibiliary diarrhea) Zof-e- } \\
\text { qalb wa me'da (weakness of heart and stomach), } \\
\text { Qai (vomiting) }(10,11,12)\end{array}$} \\
\hline Sharbat seb Sheereen (10) & - & $\begin{array}{c}2250 \\
\text { (Aab seb) }\end{array}$ & 500 & - & - & $1 / 2$ & \\
\hline Sharbat seb Sheereen (11) & - & $\begin{array}{c}2250 \\
\text { (Aab seb) }\end{array}$ & 500 & - & - & $1 / 2$ & \\
\hline Sharbat Halyun (11) & 72 & - & 480 & - & $1: 6$ & - & \multirow{2}{*}{$\begin{array}{l}\text { Hisat-e-kulya (nephrolithiasis), Hisat-e-masana } \\
\text { (bladder stone), Mudirr-e-bol } \\
\text { (diuretic) }(11,12) \text { ) }\end{array}$} \\
\hline Sharbat Halyun (12) & 135 & 1920 & 960 & $1: 14$ & $1: 7$ & - & \\
\hline Sharbat Gaozaban (12) & 225 & 960 & 900 & $1: 4$ & $1: 4$ & - & $\begin{array}{l}\text { Zo'f-e-qalb (weakness of heart), Khafqan } \\
\text { (palpitation) (12) }\end{array}$ \\
\hline Sharbat Kharkhask (12) & 240 & - & 1020 & - & $1: 4$ & - & $\begin{array}{l}\text { Hisat-e-masana (bladder stone), Zo'f-e-bah } \\
\text { (anaphrodisia) (12) }\end{array}$ \\
\hline Sharbat Mufattit (12) & 190 & - & 1080 & - & $1: 4$ & - & $\begin{array}{l}\text { Hisat-e-kulya (nephrolithiasis), Mudirr-e-bol } \\
\text { (diuretic) (12) }\end{array}$ \\
\hline Sharbat Kaknaj (12) & 231 & - & 480 & - & $1: 2$ & - & $\begin{array}{l}\text { Soozak (gonorrhea), } \\
\text { Qarh-e-masana (bladder ulcer) (12) }\end{array}$ \\
\hline Sharbat Afsanteen (12) & 140 & 1920 & 420 & $1: 14$ & $1: 3$ & $1 / 3$ & $\begin{array}{l}\text { Zo'f-e-me'da (weakness of stomach), } \\
\text { Zo'f-e-ishteha (anorexia) (12) }\end{array}$ \\
\hline Sharbat Balango (12) & 1065 & - & 960 & - & 1:1 & - & $\begin{array}{l}\text { Zof-e-qalb wa me'da (weakness of heart and } \\
\text { stomach), Malan-khuliya (melancholia) (12) }\end{array}$ \\
\hline Sharbat Izkhar (12) & 226 & 960 & 2880 & $1: 4$ & 1:10 & $1 / 2$ & $\begin{array}{l}\text { Yarqan (jaundice), Istesqa (ascites), Tasaddud-e- } \\
\text { kabid (hepatic obstruction) (12) }\end{array}$ \\
\hline Sharbat Sapistan (12) & 60 & 1920 & 960 & $1: 3$ & $1: 16$ & - & $\begin{array}{l}\text { Dard wa warm-e-halaq (pharyngeal pain), } \\
\text { Khushoonat-e-halaq (sore throat) Sual (cough) } \\
\text { (12) }\end{array}$ \\
\hline Sharbat Habbul Aas (11) & 408 & 3000 & 1000 & $1: 7$ & $1: 2.5$ & - & $\begin{array}{l}\text { Zo'f-e-me'da (weakness of stomach), Ishal } \\
\text { (diarrhea), Nazf-ud-dam (hemorrhage) (11) }\end{array}$ \\
\hline Sharbat Humaz (11) & 168 & - & 960 & - & $1: 6$ & - & $\begin{array}{l}\text { Zo'f-e-me'da wa qalb (weakness of stomach and } \\
\text { heart), Khaf-qan (palpitation) (11) }\end{array}$ \\
\hline SharbatZufa Murakkab (11) & 145 & 1000 & 500 & $1: 7$ & $1: 3$ & - & $\begin{array}{l}\text { Balghami sual (phlegmatic cough), Zeeq-un-nafas } \\
\text { (asthma)11 }\end{array}$ \\
\hline Sharbat Kewda (11) & - & $\begin{array}{l}900 \\
\text { (Arq kewda) }\end{array}$ & 900 & - & $1: 1$ & - & $\begin{array}{l}\text { Atash-e-mufrit (excessive thirst), Musakki-ne- } \\
\text { qalb (defrigent) (11) }\end{array}$ \\
\hline Sharbat Mushil (11) & 300 & - & 672 & - & $1: 2$ & - & Shadeed qabz (severe constipation) (11) \\
\hline Sharbat Mulayyan (11) & 294 & - & 900 & - & $1: 3$ & - & $\begin{array}{l}\text { Muzliq-e-am’a (lienteric diarrhea), } \\
\text { Qabz (constipation) (11) }\end{array}$ \\
\hline Sharbat Angoor Tursh (10) & - & $\begin{array}{c}900 \\
\text { (Aab angoor) }\end{array}$ & 2700 & - & $1: 3$ & - & $\begin{array}{l}\text { Zo'f-e-bah (anaphrodisia), Zo'f-e-am (general } \\
\text { weakness) }(10,11)\end{array}$ \\
\hline Sharbat Behi (10) & 500 & - & 500 & - & $1: 1$ & - & $\begin{array}{l}\text { Zo'f-e-me'da wa qalb (weakness of stomach and } \\
\text { heart), Zo-'f-e-ishteha (anorexia) (10) }\end{array}$ \\
\hline
\end{tabular}




\begin{tabular}{|c|c|c|c|c|c|c|c|}
\hline Name of the Sharbat & $\begin{array}{l}\text { Drug } \\
\text { (g) }\end{array}$ & $\begin{array}{l}\text { Water } \\
(\mathrm{mL})\end{array}$ & $\begin{array}{l}\text { Sugar } \\
\text { (g) }\end{array}$ & $\begin{array}{l}\mathrm{D} \& \mathrm{~W} \\
\text { ratio }\end{array}$ & $\begin{array}{l}\mathrm{D} \& S \\
\text { ratio }\end{array}$ & $\begin{array}{l}\text { Yield of } \\
\text { Joshanda }\end{array}$ & $\begin{array}{l}\text { Therapeutic } \\
\text { Use/Medicinal Actions }\end{array}$ \\
\hline Sharbat Belgiri (13) & 1000 & - & 4000 & - & $1: 4$ & - & Pechish (dysentery), Dast (diarrhea) (13) \\
\hline Sharbat Khaksi (13) & 764 & - & 1500 & - & $1: 2$ & - & $\begin{array}{l}\text { Judri (chickenpox), Hasba (measles), Moti Jhara } \\
\text { (typhoid), Nau-bati Hummiyat (remittent fever) (13) }\end{array}$ \\
\hline Sharbat Musaffi khoon (13) & 766 & - & 4000 & - & $1: 5$ & - & $\begin{array}{l}\text { Fasad-e-khoon (putrefaction of blood), Aatishak } \\
\text { (syphilis), Qooba (ringworm) (13) }\end{array}$ \\
\hline Sharbat Nankhowah (13) & 245 & - & 4000 & - & $1: 16$ & - & Zof-e-Ishtiha (anorexia), Kaasir Riyah (carminative) (13) \\
\hline Sharbat Salajeet (13) & 430 & - & 4000 & - & $1: 9$ & - & Jiryan (prostatorrhea), Zo'f-e-bah (anaphrodisia) (13) \\
\hline
\end{tabular}

\section{REFERENCES}

1. Said M. Hamdard pharmacopoeia of eastern medicine. Delhi: Sri Satguru Publications; p 169, 1997.

2. Department of AYUSH. National Formulary of Unani Medicine. Part-I. New Delhi: Ministry of Health and Family Welfare, Govt of India pp 221-224, 331-334 2006.

3. Williams $L$, Wilkins. Remington-The science and practice of pharmacy. Vol I, 21st ed. New Delhi: Wolters Kluwer Health Pvt. Ltd, p 754, 2982006.

4. T Lesley AC. L. The herbs of life-health. Delhi: Sri Satguru Publications, p 188, 1998.

5. (Internet) cited on October 10, 2015. Available from https:// en.wikipedia.org/wiki/Sharbat.

6. Department of AYUSH. Al-qarabadeen. 2nd ed. New Delhi: CCRUM, pp 603-604, 6492006.
7. Jurjani Ismail. Zakheera khawarzamshahi. Vol. VIII, New Delhi: Idara Kitabush Shifa, p 192010.

8. Kabeeruddin M. Bayaz kabeer Part 3. New Delhi: Idara Kitabush Shifa pp 71-77, 2010.

9. Department of AYUSH. Qarabadeene majeedi. Delhi: All India Tibbi Conference, p 196, 1986.

10. Abdul Hafeez Hkm. Qarabadeen jadeed. New Delhi: CCRUM, pp 119-131, 2005.

11. Kabeeruddin M. Bayaz kabeer. Part 2. New Delhi: Idara Kitabush Shifa, pp 80-87, 2010.

12. Khan Shareef Mohd. Ilaj-ul-amraz. New Delhi: Aijaz Publishing House, pp 63, 269-270, 399-401, 457,562-564,768, 2006.

13. Department of AYUSH. National Formulary of Unani Medicine. Part-V. New Delhi: Ministry of Health and Family Welfare, Govt of India, pp 138-142 2008. 